\title{
Transverse Oscillations for Phased Array Vector Velocity Imaging
}

Pihl, Michael Johannes; Jensen, Jørgen Arendt

Published in:

Proceedings of the 2010 IEEE International Ultrasonics Symposium

Link to article, DOI:

10.1109/ULTSYM.2010.0336

Publication date:

2010

Document Version

Early version, also known as pre-print

Link back to DTU Orbit

Citation (APA):

Pihl, M. J., \& Jensen, J. A. (2010). Transverse Oscillations for Phased Array Vector Velocity Imaging. In Proceedings of the 2010 IEEE International Ultrasonics Symposium (pp. 1323-1327). IEEE.

https://doi.org/10.1109/ULTSYM.2010.0336

\section{General rights}

Copyright and moral rights for the publications made accessible in the public portal are retained by the authors and/or other copyright owners and it is a condition of accessing publications that users recognise and abide by the legal requirements associated with these rights.

- Users may download and print one copy of any publication from the public portal for the purpose of private study or research.

- You may not further distribute the material or use it for any profit-making activity or commercial gain

- You may freely distribute the URL identifying the publication in the public portal

If you believe that this document breaches copyright please contact us providing details, and we will remove access to the work immediately and investigate your claim. 


\title{
Transverse Oscillations for Phased Array Vector Velocity Imaging
}

\author{
Michael Johannes Pihl and Jørgen Arendt Jensen \\ Center for Fast Ultrasound Imaging, Department of Electrical Engineering, \\ Technical University of Denmark, DK-2800 Kgs. Lyngby, Denmark
}

\begin{abstract}
Medical ultrasound imaging is widely used to visualize blood flow in the human circulatory system. However, conventional methods are angle dependent. The Transverse Oscillation (TO) method is able to measure the lateral velocity component, and it has been demonstrated in in vivo measurements of superficial blood vessels. To broaden the usability of the method, it should be expanded to a phased array geometry enabling vector velocity imaging of the heart. Therefore, the scan depth has to be increased to $10-15 \mathrm{~cm}$. This paper presents suitable pulse echo fields (PEF). Two lines are beamformed in receive to obtain lateral spatial in-phase and quadrature components. The relative mean bias and standard deviation of the lateral velocity component are computed as performance measures. For the PEF, the coefficient of variance, $C V$, of the spectral frequencies, and the energy ratio, $E R$, of leakage into negative frequencies are used as metrics to assess estimator performance. At $10 \mathrm{~cm}$ 's depth for an initial setup, the relative mean bias and standard deviation are $9.1 \%$ and $9.5 \%$, respectively. At a depth of $15 \mathrm{~cm}$, the values are $20 \%$ and $13 \%$, respectively. The PEF metric $E R$ can be used to assess the bias (correlation coefficient, $R$ : $\mathbf{- 0 . 7 6}$ ), and therefore predict estimator performance. $C V$ is correlated with the standard deviation $(R=0.74)$. The results demonstrate the potential for using a phased array for vector velocity imaging at larger depths, and potentially for imaging the heart.
\end{abstract}

\section{INTRODUCTION}

Medical ultrasound is widely used to image blood flow. For instance, the estimation of blood velocities plays a key role in diagnosing carotid artery stenosis [1]. However, blood velocity estimates using conventional color flow imaging or Doppler techniques are angle dependent. This poses a huge challenge for quantitatively measuring the magnitude (and direction) of the blood's velocity. Several techniques have been proposed to remedy this. The Transverse Oscillation (TO) method by Jensen and Munk [2] and the similar approach by Andersen [3] are two of them. The TO method has shown intriguing in vivo results of blood vector velocities in superficial vessels using linear arrays [4], and is a technique close to a commercial breakthrough [5]. However, the current implementation limits the scan depth, and hence, prevents imaging of deeper lying vessels and organs, e.g. the heart. To broaden its usability, the method should be expanded to phased arrays enabling vector velocity imaging of the heart. Therefore, suitable pulse-echo fields (PEFs) with transverse oscillations have to be designed for depths up to $10-15 \mathrm{~cm}$, and velocity measurements have to be performed.

The purpose of this paper is to expand the TO method to a phased array allowing blood flow measurements down to 10 to $15 \mathrm{~cm}$. The theory of the method is briefly stated in Section II including the application of the technique for a phased array. The section also includes a description of measures to evaluate the performance of the estimator and metrics to evaluate PEFs. The simulation setup is described in Section III followed by the obtained results in Section IV. The results are discussed in Section V and the conclusions stated in Section VI.

\section{THEORY}

This section describes the theoretical background of the Transverse Oscillation method for a phased array along with performance measures and pulse echo field metrics.

\section{A. Transverse Oscillation method}

In the following, the generated pressure field using a phased array is described, followed by beamforming approaches, and the velocity estimation.

1) Field generation and beamforming: The basic idea in the TO method is to create a double oscillating pulse-echo field as illustrated in Fig. 1. This is accomplished by using special apodization profiles in receive, whereas the transmitted beam is similar to the one used in conventional velocity estimation. A derivation of the required apodization functions and a description of the respective generated fields for the linear array can be found in [2], [6].

Using the Fraunhofer approximation, the relation between the lateral spatial wavelength, $\lambda_{x}$, and the apodization function is

$$
\lambda_{x}=\frac{2 \lambda_{z} z_{0}}{d}
$$

where $d$ is the distance between the two peaks in the apodization function, $z_{0}$ is depth, and $\lambda_{z}$ is the axial wavelength.

As (1) shows, the lateral wavelength increases as the depth increases, if the apodization function is kept constant ( $d$ is constant). To keep a constant lateral wavelength, the aperture must expand width depth. Using a phased array, the width is often limited, so instead the spacing between the two beamformed lines can be increased through depth. Keeping the apodization function fixed, the two lines can be beamformed with a fixed angle.

Using the tangent-relation (see Fig. 2) and (1), the angle between the two lines can be derived as

$$
\theta / 2=\arctan \frac{\lambda_{x} / 8}{z_{0}}=\arctan \frac{\lambda_{z}}{4 d} .
$$

The transverse oscillations are created in receive, and two lines are beamformed simultaneously to get the spatial lateral 


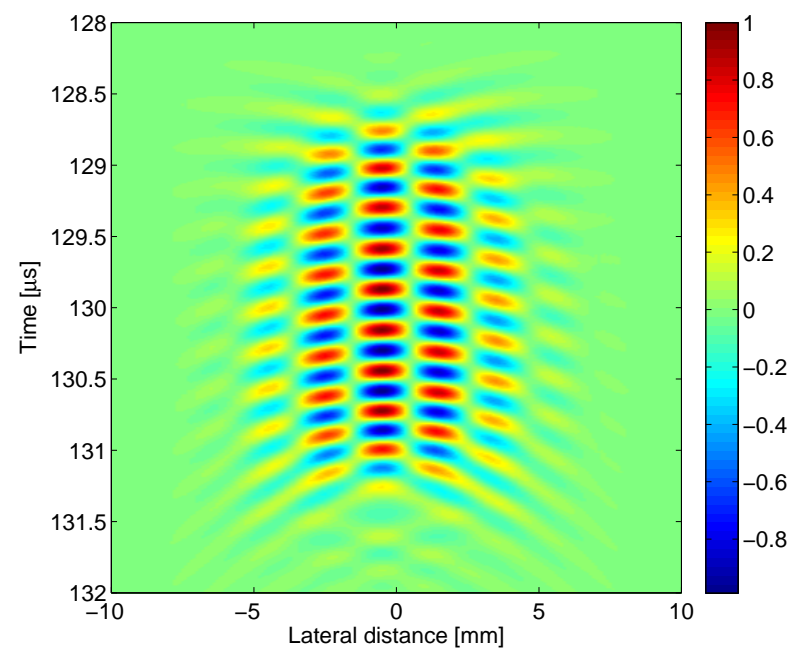

Fig. 1. Pulse-echo field for the left beam in normalized pressure for the reference simulation point at a depth of $10 \mathrm{~cm}$.

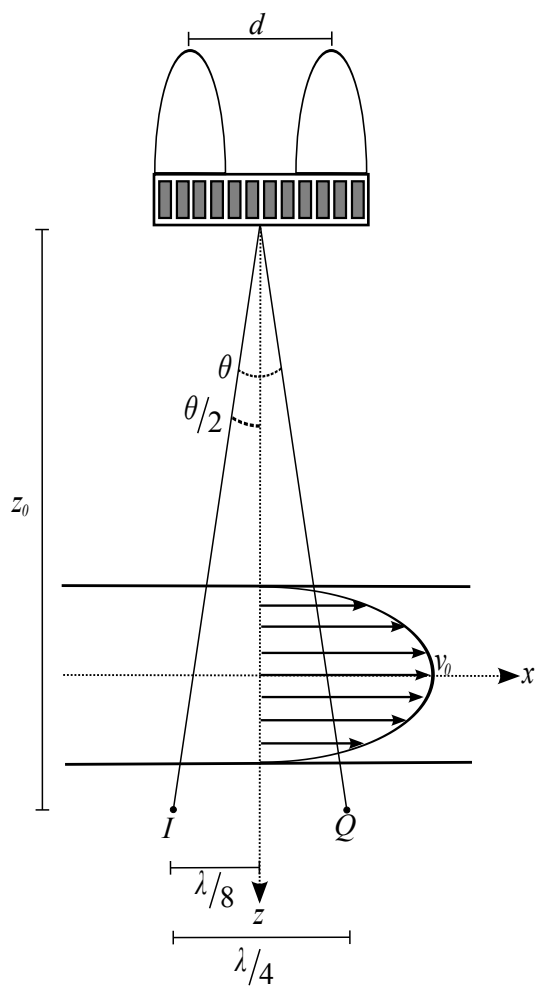

Fig. 2. Beamforming approach based on a fixed aperture function with a fixed angle between beamformed lines corresponding to the increasing spatial lateral wavelength over depth. The location of the simulation phantom with a parabolic flow profile and peak velocity, $v_{0}$, is also indicated.

in-phase (I) and quadrature (Q) components (left and right beam on Fig. 2). The spatial IQ samples, $r_{I Q}$, are obtained by

$$
r_{I Q}=r_{I}+j r_{Q}
$$

where $r_{I}$ and $r_{Q}$ are the samples from the left and right beam, respectively.

For a spacing of $20 \mathrm{~mm}$ of the apodization peaks, a center frequency of $3.5 \mathrm{MHz}$ and $c=1540 \mathrm{~m} / \mathrm{s}$, the angle between the two lines will be $0.63^{\circ}$. At a depth of $10 \mathrm{~cm}$ this corresponds

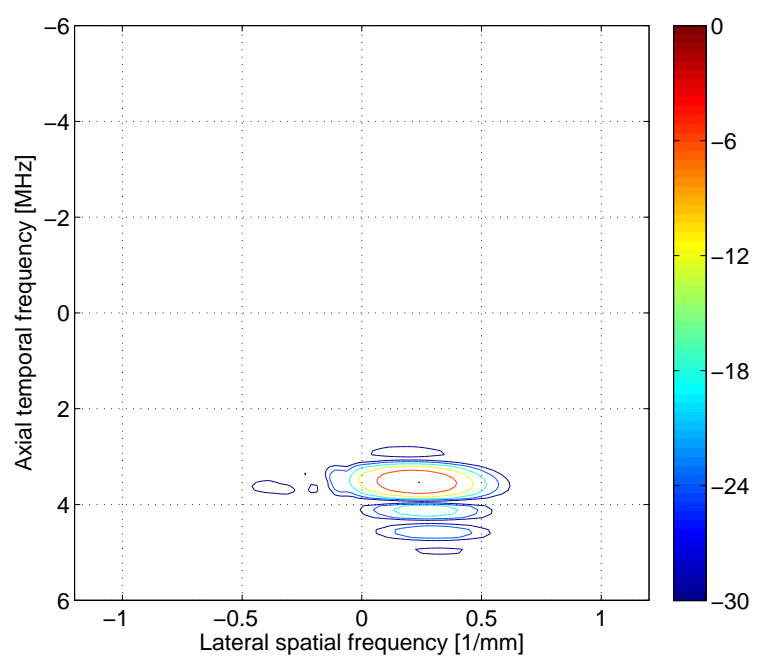

Fig. 3. The spatio-temporal frequency spectrum ( $k$-space) for the spatiotemporal IQ pulse-echo field. Shown as a contour plot of the normalized amplitude spectrum with $6 \mathrm{~dB}$ between contours. Note that the spectrum's energy mainly is located in one quadrant.

to $\lambda_{x} / 4=1.1 \mathrm{~mm}$. The $\lambda_{x} / 4$ spacing results in spatial IQ sampling for the particular modulation frequency.

Along with the two TO lines, a center line can be beamformed for conventional axial velocity estimation.

2) Velocity estimation: For a description and derivation of the estimator used for subsequent calculations, the reader is referred to [7]. Prior to the velocity estimation, the data are matched filtered by convolving with the time inverted excitation pulse. This is followed by stationary echo canceling, where the mean of $N$ emitted signals is subtracted from each emission. No discrimination has been performed to distinguish between the stationary and the moving scatterers.

\section{B. Statistical performance measures}

To investigate the performance of the method, a statistical analysis is performed on the simulated data. It is assumed that the velocity estimates are independent.

At each discrete depth in the vessel, the velocity is estimated from a number of emissions. The mean, $\bar{v}\left(z_{k}\right)$, of $N$ estimates and the estimated standard deviation, $\sigma\left(z_{k}\right)$, is calculated at each discrete depth.

For better and more straightforward comparison of various parameter settings, two single measures for the bias and the estimated standard deviation for a specific velocity profile are computed. In order to do so, the estimated variance and bias are averaged over the entire vessel and divided by the peak velocity, $v_{0}$. The two quantities, the relative mean bias, $\tilde{B}$, and the estimated relative mean standard deviation, $\tilde{\sigma}$, are given by

$$
\begin{aligned}
\tilde{B} & =\frac{1}{v_{0} N_{z_{k}}} \sum_{z_{k}=1}^{N_{z_{k}}} B\left(z_{k}\right) \\
\tilde{\sigma} & =\frac{1}{v_{0}} \sqrt{\frac{1}{N_{z_{k}}} \sum_{z_{k}=1}^{N_{z_{k}}} \sigma\left(z_{k}\right)^{2}},
\end{aligned}
$$


where $N_{z_{k}}$ is the number of discrete samples within the vessel. These measures can to some extend be used to describe the performance of the TO estimator.

\section{PEF metrics}

The simulations take a long time to perform to obtain enough estimates for evaluating the performance of the estimator. Therefore, it would be desirable if the performance to some extend could be predicted from the spatio-temporal frequency spectrum. Two metrics are suggested as described in the following.

Ideally, the lateral spatial frequencies of the two added signals should yield a narrow one-sided spectrum (see Fig. 3) in the lateral dimension of the 2D spatio-temporal spectrum. Using temporal IQ sampled data, or the Hilbert transform, the spectrum will already be one-sided in the axial dimension.

The mean spatial frequency depends on the aperture function, i.e. the spacing of the two apodization peaks. The width of the apodization peaks determine the frequency spread in the spatio-temporal spectrum, $S(f)$.

One of the metrics used to evaluate the designed PEFs is the coefficient of variation, $C V$, of the spectral frequencies

$$
C V=\frac{\sigma_{f x}}{\bar{f}_{x}}=\frac{\sqrt{\frac{\int_{-\infty}^{\infty}\left(f_{x}-\bar{f}_{x}\right)^{2} S\left(f_{x}, f_{0}\right)^{2} d f_{x}}{\int_{-\infty}^{\infty} S\left(f_{x}, f_{0}\right)^{2} d f_{x}}}}{\frac{\int_{-\infty}^{\infty} f_{x} S\left(f_{x}, f_{0}\right)^{2} d f_{x}}{\int_{-\infty}^{\infty} S\left(f_{x}, f_{0}\right)^{2} d f_{x}}}
$$

where $C V$ is computed at the (temporal) center frequency, $f_{0}, \bar{f}_{x}$ is the mean spectral (lateral) frequency, and $\sigma_{f_{x}}$ is the spatial standard deviation. It is hypothesized that this terms is related to the standard deviation of the velocity estimates.

Furthermore, the estimated mean spatial frequency can be used in the estimator to yield a more unbiased estimate. This is due to the fact that the Fraunhofer approximation is not $100 \%$ valid.

The second metric is the energy ratio, $E R$, computed as

$$
E R=\frac{\int_{-\infty}^{0} S\left(f_{x}, f_{0}\right)^{2} d f_{x}}{\int_{-\infty}^{\infty} S\left(f_{x}, f_{0}\right)^{2} d f_{x}}
$$

at the center frequency, $f_{0}$. This provides information of the energy leak into the other half of the spatio-temporal frequency spectrum, e.g. looking at Fig. 3 from positive spatial frequencies to negative. It is hypothesized that this gives an estimate of the bias of the velocity profiles. When the value of $E R$ is $50 \%$ the energy is evenly distributed between both quadrants, and velocity estimation is expected to be compromised.

\section{Simulation SETUP}

The ultrasound simulation program Field II [8], [9] was used for the simulation study. A phased array transducer with the characteristics shown in Table I was emulated. A blood vessel (as illustrated in Fig. 2) was modeled as a cylindrical volume (radius $=6 \mathrm{~mm}$ ) of moving scatterers surrounded by a block of stationary scatters in a $2 \times 2 \times 2 \mathrm{~cm}$ scatter block. The number of
TABLE I

Simulation SETUP

\begin{tabular}{ll}
\hline Transducer parameter & Value \\
\hline Transducer & Phased array \\
Number of elements & 128 \\
Pitch & $0.220 \mathrm{~mm}$ \\
Kerf & $0.022 \mathrm{~mm}$ \\
Center frequency & $3.5 \mathrm{MHz}$ \\
Sampling frequency & $100 \mathrm{MHz}$ \\
\hline Fixed simulation parameter & Value \\
\hline Pulse repetition frequency & $5 \mathrm{kHz}$ \\
Speed of sound & $1540 \mathrm{~m} / \mathrm{s}$ \\
Maximum velocity of blood & $1.0 \mathrm{~m} / \mathrm{s}$ \\
No. of transmit cycles in pulse & 8 \\
Transmit focus (radial depth) & $10 \mathrm{~cm}$ \\
Signal-to-noise ratio (SNR) & $\infty$ \\
\hline
\end{tabular}

TABLE II

VARIED SIMULATION PARAMETERS

\begin{tabular}{ll}
\hline Parameter & Value \\
\hline Center of vessel & $234.57 .5 \mathbf{1 0} 15\left[^{\circ}\right.$ ] \\
Scan angle & $-30-15 \mathbf{0 1 5} 30$ [degrees] \\
F\# in transmit & $468 \mathbf{1 0} 12$ \\
Transmit apodization shape & Hanning, Tukey, Rectangular \\
Receive apodization shapes & Hanning, Tukey, Rectangular \\
Space between peaks & $164880 \mathbf{9 6} 112$ \\
Apodization width & $816 \mathbf{3 2} 64$ \\
Number of shots per estimate & $4816 \mathbf{3 2} 64$ \\
Transverse lag in estimator & $\mathbf{1} 345$ \\
&
\end{tabular}

scatterers was 6726 to obtain 10 scatterers per resolution cell. The flow angle was set to be perpendicular to the depth axis to obtain purely transverse flow at a scan angle of 0 degrees. When scanning at angles different from zero the scatter block was translated to the new position (not rotated).

The fixed simulation parameters are listed in Table I as well. To investigate the performance under optimal conditions, no noisy was added to the simulated data, hence the SNR of $\infty$.

Table II lists the parameters that where varied in the simulation study. A number of initial conditions were used to form a starting point in parameter space as indicated with bold face in Table II. During this study, the different parameters were kept fixed at their starting point (unless otherwise stated), and only the parameter under investigation was varied. Every simulation setup was repeated 20 times.

For the apodization shapes, the Hanning, the Tukey, and the rectangular shapes can be modeled as Tukey windows with ratio of tapers as $1,0.5$, and 0 , respectively. When testing the space between the receive apodization peaks, the apodization width was set to 16 elements instead of 32 elements as in the rest of the study in order to increase the possible spacing. At a depth of $10 \mathrm{~cm}$ these spacings, [16 488096 112] elements, correspond to $\lambda_{x}=\left[\begin{array}{lllll}25 & 8.3 & 5.0 & 4.2 & 3.6\end{array}\right] \mathrm{mm}$. When testing the width of the receive apodization peaks, the spacing between the peaks was set to 64 instead of the 96 in the rest of the study, as there is only 128 elements in the transducer.

The flow was modeled to be laminar with a parabolic flow profile (see Fig. 2) given by

$$
v(r)=\left(1-\frac{r^{2}}{R^{2}}\right) v_{0},
$$



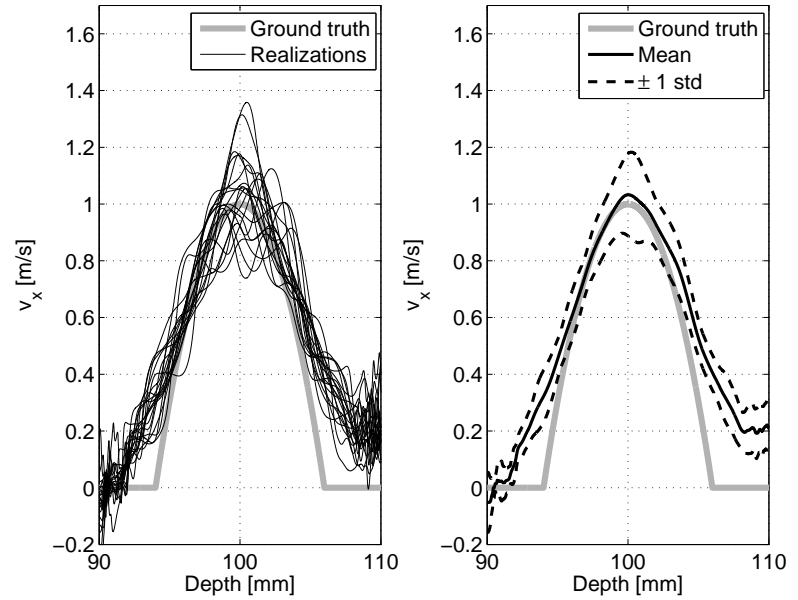

Fig. 4. [Left] Transverse velocity estimates from 20 realizations. [Right] the mean estimate plus/minus one standard deviation. Note that no effort has been made to remove the estimates outside the vessel

where $r$ denotes the radial position, $R$ is the radius of the tube, and $v_{0}$ is the maximum velocity at the center of the tube.

The beamformed data are matched filtered and mean stationary echo canceling is performed prior to velocity estimation as previously described.

\section{RESULTS}

\section{A. Initial point}

Initially, the simulation phantom is positioned at a (radial) depth of $10 \mathrm{~cm}$ at a scan angle of $0^{\circ}$. The transmit apodization of the transducer is a Hanning function with a width corresponding to a F-number of 10 . The two receive apodization shapes are two Hanning functions with widths of 32 elements $(7.0 \mathrm{~mm})$ spaced 96 elements $(21 \mathrm{~mm})$ apart. The number of shots per estimate, $N$, and the transverse lag, $k$, are parameters that only affect the estimator itself, and they are 32 and 1, respectively.

The lateral velocity estimates, $v_{x}$, from the 20 realizations are shown in Fig. 4 on the left. The 20 realizations closely follow the ground truth, as illustrated on the right panel showing the mean of the 20 realizations plus/minus one standard deviation. With these settings the relative mean bias, $\tilde{B}_{v_{x}}$, is $9.1 \%$ and the relative standard deviation, $\tilde{\sigma}_{v_{x}}$, is $9.5 \%$.

\section{B. Parameter study}

1) Physical parameters: The effect on performance measures when changing the position of the phantom in terms of (radial) scan depth and scan angle is illustrated in Fig. 5. $\tilde{B}_{v_{x}}$ is closet to zero at a depth of $7.5 \mathrm{~cm}$. With the given setup, the estimator underestimates velocity at low depths, and overestimates at large depths. $\tilde{\sigma}_{v_{x}}$ increases with depth. For $\tilde{B}_{v_{x}}$, the performance decreases when steering the beam away from $0^{\circ}$.

2) Transmit parameters: On the transmit side, the Fnumber and the shape of the apodization has been varied. There is no real difference between the performance measures for the different transmit types. For the given setup, the most
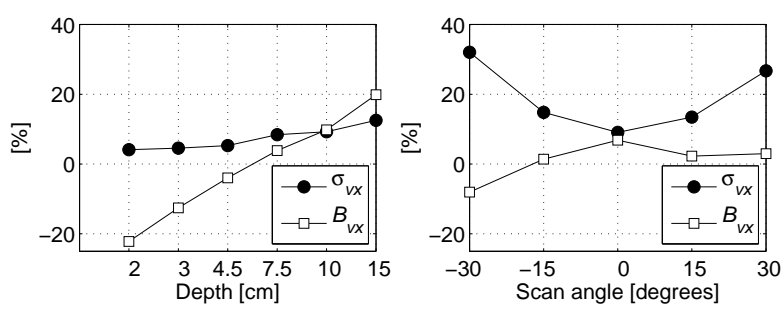

Fig. 5. Performance measures for different scan depths and scan angles.
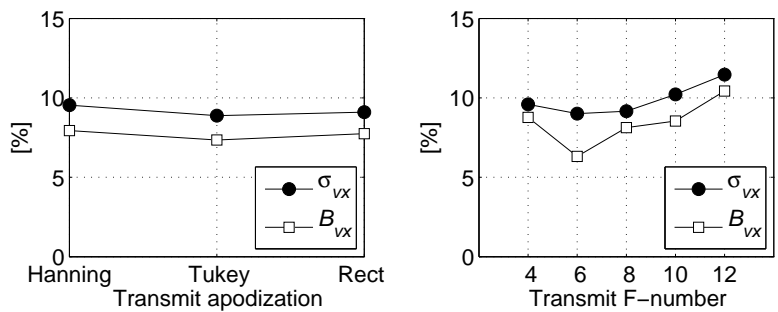

Fig. 6. Performance measures for transmit apodization type and F-number.
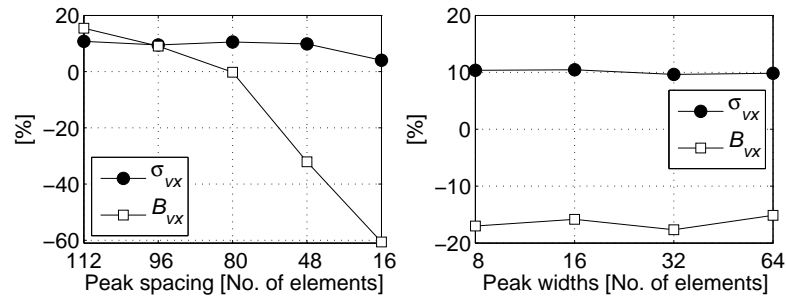

Fig. 7. Performance measure for receive apodization peak spacing and width.
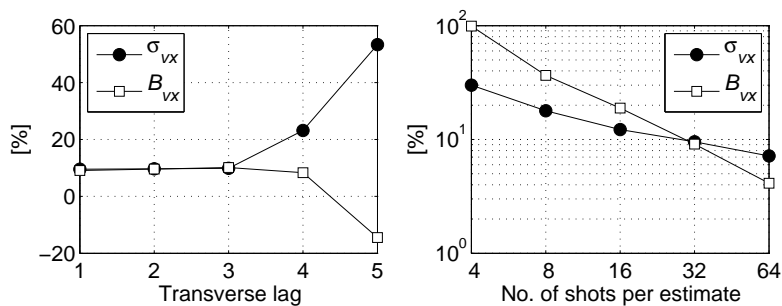

Fig. 8. Performance measures for transverse lag and no. of shots per estimate.
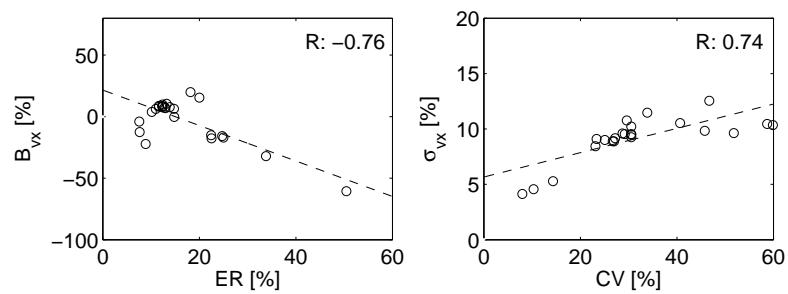

Fig. 9. Scatter plot of PEF metrics and performance measures for $E R \&$ $\tilde{B}_{v_{x}}$ [left] and $C V \& \tilde{\sigma}_{v_{x}}$ [right]. The correlation values are -0.76 , and 0.74 , respectively.

accurate and precise velocities estimates are obtained with a F-number of 6 as shown in Fig. 6.

3) Receive parameters: As mentioned, the receive apodization creates the transverse oscillations in the pulse-echo field. The result of changing apodization functions, width, and position is depicted in Fig. 7. Decreasing the peak spacing below a certain point yields a significant degradation of $\tilde{B}_{v_{x}}$, illustrating that the estimator gets increasingly biased, and is 
unable to estimate the velocity accurately. However, $\tilde{\sigma}_{v_{x}}$ is not affected. For the given reference setup (at $10 \mathrm{~cm}$ 's depth), changing the width of the peak has no influence on the two performance measures. Using different receive types yielded the same trends as for the transmit case (data not shown).

4) Estimator parameters: As Fig. 8 illustrates, increasing the number of shots per estimate decreases $\tilde{\sigma}_{v_{x}}$ with approximately the expected $1 / \sqrt{N}$. Also $\tilde{B}_{v_{x}}$ decreases. The transverse lag can be increased to $k=3$ without affecting performance, thereafter aliasing increasingly occurs.

\section{Performance measures}

To test the hypothesis whether there are a correlation between the PEF metrics and performance measures, the values are plotted in Fig. 9. The correlation coefficients are -0.76 for $E R$ and $\tilde{B}_{v_{x}}$. For $C V \& \tilde{\sigma}_{v_{x}}$ the value is 0.74 . In both cases, the values indicate that there is a correlation. For the latter, 2 outliers have been removed. The criteria is the energy ratio, i.e. the energy leak. The cut-off is set remove data points where $E R>33 \%$. When $50 \%$, the energy is located in both halves of the spectrum. A value slightly lower than this is selected to ensure that energy leak into the other quadrant is limited, so that the velocity is estimated without too much bias.

The correlation coefficients indicate that there is a correlation between the PEF metrics and the performance measures. Hence, the PEF metrics can be used as an initial assessment of the expected estimator performance. The PEF metrics can be readily computed opposed to the simulated velocity data and the derived performance measures.

\section{Discussion}

As the depth increases, the transverse spatial frequency decreases and thereby affecting the precision of the estimator. The bias is negative at low depths and positive at large depths for the current setup. A more unbiased estimate might be obtained, if the actual spatial wavelength was used and not the theoretically derived one, either by estimating it or deriving it from the pulse-echo field. Furthermore, training the estimator on data set could provide bias correction terms for different setups. The underlying assumption is that the bias is constant and deterministic for a given spatial location with a specific setup.

There are some performance issues at increasing steering angle, but they might be resolved with further optimization of the pulse-echo field taking the increasing steering angle under consideration.

It can be noted that the transmit F-number can be optimized for the depth. The apodization types (transmit and receive) did not significantly affect performance. However, the different combinations should be investigated, especially having two rectangular apodizations. The larger the area under the apodization functions, the better SNR can be obtained in a noisy environment.

Performance decreases when peak separation in receive decreases, due to a lower spatial frequency. Additionally, a poorer spatial resolution is obtained. The apodization width affects $C V$, however, does not seem to affect $\tilde{\sigma}_{v_{x}}$.
Because of the decreasing spatial frequency through depth, the velocity range increases with depth. However, the estimator appears to be unaffected within its velocity range.

The PEF metric $E R$ is correlated with $\tilde{B}_{v_{x}}$ and can be used to predict when the performance of the estimator will deteriorate due to a poor PEF and corresponding spatiotemporal spectrum. $C V$ can to some extend give a hint of the magnitude of $\tilde{\sigma}_{v_{x}}$.

Overall, the estimator is not very sensitive to small changes in parameter settings. In some cases the performance degrades in the sense that the velocities are not estimated correctly, however, this is done consistently as the standard deviation is not affected. In some cases, the degradation can be predicted by calculating $E R$ of the PEF. In general, a lot can be learned by investigating the PEF prior to simulation and optimizing it.

\section{CONCLUSION}

Various parameters have been investigated around a reference point at $10 \mathrm{~cm}$ 's depth where $\tilde{B}_{v_{x}}$ is $9.1 \%$ and $\tilde{\sigma}_{v_{x}}$ is $9.5 \%$. Overall, the estimator is not very sensitive to minor changes in the setup. At a depth of $15 \mathrm{~cm}$, it was possible to estimate the lateral velocity with $\tilde{B}_{v_{x}}$ and $\tilde{\sigma}_{v_{x}}$ being $20 \%$ and $13 \%$, respectively. This demonstrates that in simulation without noise, the velocity can be estimated at $10-15 \mathrm{~cm}$ 's depth with standard deviations on par with conventional estimators.

The results are encouraging for further development and for obtaining measured velocity data. And potentially, for imaging the heart with phased array vector velocity imaging.

\section{ACKNOWLEDGMENTS}

This work was supported by grant 024-2008-3 from the Danish Advanced Technology Foundation and BK Medical Aps, Denmark.

\section{REFERENCES}

[1] E. G. Grant et al., "Carotid artery stenosis: Gray-scale and doppler us diagnosis - society of radiologists in ultrasound consensus conference," Radiology, vol. 229, no. 2, pp. 340-346, 2003.

[2] J. A. Jensen and P. Munk, "A New Method for Estimation of Velocity Vectors," IEEE Trans. Ultrason., Ferroelec., Freq. Contr., vol. 45, pp. 837-851, 1998.

[3] M. E. Anderson, "Multi-dimensional velocity estimation with ultrasound using spatial quadrature," IEEE Trans. Ultrason., Ferroelec., Freq. Contr., vol. 45, pp. 852-861, 1998.

[4] K. L. Hansen, J. Udesen, C. Thomsen, J. A. Jensen, and M. B. Nielsen, "In-vivo validation of transverse oscillation vector velocity estimation with MR angiography," IEEE Trans. Ultrason., Ferroelec., Freq. Contr., vol. 56, no. 1, pp. 91-100, 2009.

[5] M. J. Pihl, S. Nikolov, P. Haugaard, M. C. Hemmsen, and J. A. Jensen, "Performance of the transverse oscillation method using beamformed data from a commercial scanner," in Proc. IEEE Ultrason. Symp., 2009, p. Accepted.

[6] J. Udesen and J. A. Jensen, "Investigation of Transverse Oscillation Method," IEEE Trans. Ultrason., Ferroelec., Freq. Contr., vol. 53, pp. 959-971, 2006.

[7] J. A. Jensen, "A New Estimator for Vector Velocity Estimation," IEEE Trans. Ultrason., Ferroelec., Freq. Contr., vol. 48, no. 4, pp. 886-894, 2001.

[8] J. A. Jensen, "Field: A Program for Simulating Ultrasound Systems," Med. Biol. Eng. Comp., vol. 10th Nordic-Baltic Conference on Biomedical Imaging, Vol. 4, Supplement 1, Part 1, pp. 351-353, 1996.

[9] J. A. Jensen and N. B. Svendsen, "Calculation of Pressure Fields from Arbitrarily Shaped, Apodized, and Excited Ultrasound Transducers," IEEE Trans. Ultrason., Ferroelec., Freq. Contr., vol. 39, pp. 262-267, 1992. 